[Vicino Oriente XX (2016), pp. 17-28]

\title{
GLI AVVERBI DI LUOGO ITTITI IN -an: FORME DI NOMINATIVO ACCUSATIVO NEUTRO SINGOLARE?
}

\author{
Rita Francia - Sapienza Università di Roma
}

\begin{abstract}
Some scholars considered O(ld) H(ittite) local adverbs andan, āppan, kattan, peran, šer to be nom(inative) acc(usative) n(euter) s(ingular) substantives. To prove this, they provided some syntactic occurrences, in which these local adverbs would be constructed as nom. acc. n. s. nouns. This hypothesis is implausible, because these local adverbs have locative function («where?»), not directive («to what place?») in $\mathrm{OH}$, as the accusative case (accusative of direction) with motion verbs.
\end{abstract}

Keywords: local adverbs; possessive pronouns, «formans» -an; accusative of direction; directive case; locative case.

\section{STATUS QUAESTIONIS}

Nello studio della sintassi e della morfologia degli avverbi di luogo ittiti «corrispondenti» ${ }^{1}$ anda(n), āppa(n), katta(n), peran / parā e šer / šarā nella lingua antica, l'opera di Frank Starke, Die Funktionen der dimensionalen Kasus und Adverbien im Althethitischen (Studien zu den Bogazköy-Texten 23) Wiesbaden 1977, ha svolto un ruolo di primaria importanza. Riguardo alla funzione di questi avverbi, lo studioso ha evidenziato che le forme terminanti in -an e šer assolvono all'espressione dello stato, da cui la definizione di «lokativische Adverbien» ${ }^{2}$, mentre in quelle in - $a$ all'espressione della direzione, da cui la denominazione «terminativische Adverbien». Negli anni successivi molti sono stati gli studi che hanno cercato di indagare ulteriormente la morfologia e soprattutto la sintassi di questa categoria avverbiale, tra cui ci limitiamo a citare: Jacqueline Boley (1985a; 1985b), Françoise A. Tjerkstra (1999), Donna Salisbury (1999), Rita Francia (2002). In questo nostro studio abbiamo preferito definire gli avverbi in -an come «adirezionali» ${ }^{3}$ e i corrispondenti in $-a$ come «direttivi» ${ }^{4}$, terminologia che utilizzeremo d'ora in avanti anche nel presente contributo.

È convinzione di F. Starke che nella lingua antica queste forme siano ancora funzionanti come temi nominali in - $a$ - e, pertanto, siano declinate nel caso dir(ettivo) per l'espressione della direzione, e al nom(inativo)-acc(usativo) n(eutro) sing(olare), con desinenza - $n$, per

1 Starke 1977, 34 «korrespondierende Adverbien», così definiti per il fatto di ricorrere sia nella forma «locativa» in -an che «terminativa» in - $a$. Le abbreviazioni bibliografiche e le sigle sono conformi a quelle adottate da Güterbock-Hoffner 1980- = CHD.

2 Accanto a questi sono da considerarsi anche $k a t t a_{2}$, formalmente simile al «terminativo» katta ma sintatticamente accostabile alla corrispondente forma «locativa» kattan, e il «locativo» katti- attestato unicamente in unione alle forme pronominali enclitiche in -i: katti-mi, -ši, -šmi, etc., Starke 1977, 181 e segg., Francia 1996a, 252 e segg.; 2002, 46 et passim.

3 Francia 2002, 22 e segg.

4 Francia 2002, 113 e segg.; per questa definizione accettiamo le motivazioni avanzate da Brixhe 1979,65 e segg.

ISSN 0393-0300

e-ISSN 2532-5159 
l'espressione dello stato ${ }^{5}$. A questo modello sfugge andan, ricondotto tradizionalmente all'i.e. *en-dom, analogamente al gr. $\varepsilon\left[v \delta o v{ }^{6}\right.$. Partendo da queste premesse, la funzione direttiva delle forme in - $a$ non necessita di altre spiegazioni per essere giustificata; più problematico, invece, risulta riconoscere una funzione stativa a nomi in caso nom.-acc. n. sing. Le funzioni sintattiche che il caso accusativo svolge in $\mathrm{O}(\mathrm{ld}) \mathrm{H}$ (ittite) in dipendenza da un verbo di moto non sono riconducibili in alcun caso ad una stasi o ad una adirezionalità ma, al contrario, all'espressione della direzione ${ }^{7} \mathrm{e}$, in aggiunta, dell'estensione nello spazio e nel tempo ${ }^{8}$.

Le ragioni per cui F. Starke ha analizzato le forme stative appan, kattan, peran come nominativi accusativi neutri singolari fanno capo a considerazioni di natura morfologica e sintattica. Da un punto di vista morfologico, infatti, essi, come già accennato, sono del tutto simili al nom.-acc. n. sing. dei temi in -a- al pari, ad esempio, di pedan; dal punto di vista sintattico, in $\mathrm{OH}$ questi avverbi occorrono nelle seguenti costruzioni:

(1) precedono un nome in caso in -i (dat[ivo]loc[ativo ${ }^{9}$ : andan É-ri);

(2) seguono un nome in caso in -i (dat.loc.: É-ri andan; LUGAL-i peran);

(3) seguono un nome in caso gen(itivo) (LUGAL-waš peran);

(4) costruiscono con forme enclitiche pronominali con uscita in -t (-met, -tet, -šet etc.) riconosciute essere pronomi possessivi enclitici in caso nom.-acc. n. sing. ${ }^{10}$.

Sull'interpretazione delle prime due costruzioni non vi è unanimità di consensi. Relativamente alla tipologia (1) (avverbio adirezionale - N[ome] dat.loc.), F. Starke ritiene sia da vedersi una costruzione appositiva ${ }^{11}$, mentre considera i sintagmi tipo (2) (N loc.dat. - avverbio adirezionale) e (3) (N gen. - avverbio adirezionale) tra loro corrispondenti da un punto di vista funzionale e paragona la tipologia (2) alla apposizione partitiva, unica costruzione ad essere in alternanza a quella genetivale nei testi antichi ${ }^{12}$. Lo studioso, inoltre, osserva che in $\mathrm{OH}$ vi è una netta prevalenza della costruzione di tipo (3) ( $\mathrm{N}$ gen. avverbio adirezionale), mentre nelle fasi successive è lo schema di tipo (2) (N dat.loc.-

5 Starke 1977, 133, 149, 172 e segg. Contrariamente a tale teoria si sono espressi Josephson 1981, 95 e segg.; Boley 1985b, 5 e segg.; Kammenhuber 1979, 115 e segg.; Francia 1996a, 221 e segg.; 2002, passim; Salisbury 1999, 61-62; Hoffner - Melchert 2008, 300.

$6 \quad$ Citiamo a titolo esemplificativo Starke 1977, 133 nota 10; Boley 1985a, 230; Melchert 1994, 135; Rieken 1999, 151. Recentemente Oettinger (2016, 232-233) ha proposto una diversa etimologia per questo avverbio, argomento su cui torneremo nel corso della presente trattazione.

7 Neu 1974, 67-68; Francia 2008, 105

8 Francia 1997; 2008, 106.

9 Starke $(1977,63-68)$ ritiene che nella lingua antica il caso locativo (desinenza -i) sia distinto dal dativo (desinenza -i), e che con gli avverbi stativi possano costruire solo nomi in caso loc.; diversamente Francia 2012, 103-104, con bibliografia precedente.

10 Si veda anche Francia 2002. Le costruzioni (3) e (4), come pure quella con il caso dat. (2) non sono documentate con andan; la costruzione (4) non è attestata con āppan nella lingua antica.

11 Starke 1977, 175: «Wie bei anda parna so liegt auch bei andan É-ri, kattan INA É halentu, šer dIŠKUR-aš É-ri eine Apposition vor, denn obwohl in der Endung formal unterschieden, nehmen lok. Adverb und Lokativ dieselbe semantische Position ein, was bedeutet, daß auch dieselbe dimensionale Funktion haben, was wiederum Voraussetzung für das Zustandekommen der Apposition ist»»; Starke 1977, 172-173.

12 «Jedes dimensionale Adveb drückt nun auf Grund seines besonderen Begriffsinhalts [....] einen Teilbegriff gegenüber dem Substantiv im dimensionalen Kasus aus». 
avverbio adirezionale), a prevalere, fino alla totale sparizione di (3) ${ }^{13}$. A conferma che la costruzione di tipo (2) (N dat.loc.- avverbio adirezionale) sia da paragonarsi ad un'apposizione partitiva, F. Starke avanza le osservazioni già formulate a suo tempo da Onofrio Carruba, Vladimir Souček, Robert Sternemann proprio relativamente all'occorrenza della costruzione con il caso gen. rispetto all'apposizione partitiva nei manoscritti delle Leggi: la prima è tipica delle redazioni più antiche, mentre la seconda di quelle posteriori ${ }^{14}$.

Riguardo alla cronologia delle due costruzioni, con gen. e con dat. loc., relativamente agli avverbi adirezionali, Erich Neu ha dimostrato che esse sono da ascriversi a due stadi linguistici differenti: $\mathrm{N}$ gen. - avverbio adirezionale, tipologia (3), è tipica di una fase linguistica più antica, mentre dat.loc. - avverbio adirezionale, tipologia (2), è più recente ${ }^{15}$. Questa evoluzione è confermata dall'affermarsi del caso dat.loc. in relazione agli avverbi adirezionali, a spese del gen., nella lingua posteriore all'antica ${ }^{16}$. Controbattendo la proposta di F. Starke, E. Neu osserva che una costruzione del tipo *peran LUGAL-i, corrispondente alla tipologia (1) (avverbio adirezionale - $\mathrm{N}$ dat.loc.) di fatto non è attestata in $\mathrm{OH}$, mentre lo sono quella di tipo (3), LUGAL-waš peran ( $\mathrm{N}$ gen. - avverbio adirezionale) e di tipo (2), LUGAL-i peran (N dat.loc. - avverbio adirezionale). Riguardo alla teoria che vede in LUGAL-i peran ( $\mathrm{N}$ dat.loc. - avverbio adirezionale) un'apposizione partitiva, Neu rimarca che, se così fosse, essa dovrebbe risultare non quale è, ma *LUGALun peran ( $\mathrm{N}$ acc. - avverbio), dal momento che peran, come tutti gli avverbi adirezionali in -an, è una forma di nom. acc. n. sing.

Da parte nostra, aggiungiamo che anche la presunta relazione appositiva nella costruzione (1) (avverbio adirezionale - $\mathrm{N}$ dat.loc.) dà adito a dubbi: se questa spiegazione è valida per andan É-ri, attestato in StBoT 25 n. 27, Vo! 13, ${ }^{17}$ e in KBo VI 2, Vo. IV 54 (LH, § 98) ${ }^{18}$, dovrebbe esserlo anche per kattan INA É halentu (avverbio adirezionale - N dat.loc.) di StBoT 25 n. 14, Vo. III 6'. Tuttavia, in quanto avverbio in -an, kattan è un nom. acc. n. sing., e dunque ci si aspetterebbe di trovare anche in questo caso una costruzione appositiva coerente che dovrebbe corrispondere a *kattan É halent $(i) \bar{u}$, con $\mathrm{N}$ in caso nom. acc. n. sing., conformemente all'avverbio, e non in caso dat.loc., come invece è di fatto documentata ${ }^{19}$.

\section{RIFLESSIONE SULLE PROBLEMATICHE ILLUSTRATE}

Da quanto è emerso fino ad ora, vi sono delle evidenti difficoltà nel considerare āppan, kattan, peran forme di nom. acc. n. sg., ci chiediamo, pertanto, se non sia possibile ricercare un'altra spiegazione per chiarire la morfologia di questi avverbi tenendo conto

3 Starke 1977, 76.

14 Carruba - Souček - Sternemann 1965, 14 e segg.

15 Neu 1974, 68

16 Queste considerazioni sono poi riprese e ribadite ulteriormente da Neu 1980a, 23 nota 48; sull'argomento anche Francia 2002, 80-81.

17 Neu 1980b.

18 Hoffner 1997.

19 La difficoltà di conciliare la morfologia di nom. acc. n. sing. degli avverbi in -an con la funzione semantica di locativo è espressa anche da Dunkel 1992, 159. 
della loro sintassi e della loro semantica. In tutte le attestazioni nella lingua antica gli avverbi in -an, usati in contesti con semantica locale ${ }^{20}$, indicano lo spazio in cui ha luogo un evento e la loro funzione è adirezionale, come illustrato dagli esempi che seguono:

(a) StBoT 25 n. 27, Vo!' 16’ [(wa-a-tar DUG te-eš-šum-mi-ya)] (17’) ${ }^{\lceil}$la 7 -hu-a-an an-da-

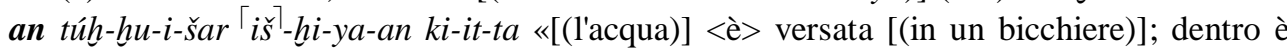
disciolto (lett. legato) incenso»;

(b) StBoT $17^{21}$, Vo. 10' I-NA MU III ${ }^{\mathrm{KAM}}$ LUGAL-uš pa-it $\left.{ }^{\mathrm{URU}} \mathrm{Za-al-pa-an} a-r a\right\rceil-a h-z a-$ an-da ú-e-te-et (11') MU II ${ }^{\mathrm{KAM}}$ kat-ta-an e-eš-ta «nel terzo anno il re andò a circondare Zalpa; per due anni $<$ le $>$ fu sotto»;

(c) StBoT 25 n. 14, Vo. III 3' [(穸e-er 'IM-aš $\square$ É-ri X NI)NDA.(ERÍN ${ }^{M E S ̌ ~ X X-i s ̌)] ~ i s ̌-t a-~}$ na-na-aš pé-e[(-ra-an $\left.\left.{ }^{\lceil} t{ }^{\top}-a n-z i\right)\right]\left(4^{\prime}\right)$ [( ${ }^{{ }^{N I N D A}}$ wa-ge-eš-šar X-li IX! NINDA.ERÍN ${ }^{M E S ̌ ~ X X-~}$

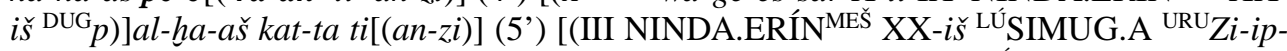

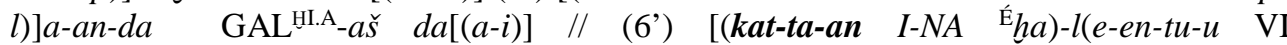
NINDA.ERÍN $\left.\left.{ }^{\mathrm{MES}} \mathrm{x}\right)\right]$ X-iš I ${ }^{\mathrm{NINDA}}$ tu-ni-ik L-iš (7’)[(( I $\left.{ }^{\rceil ?}{ }^{\mathrm{NINDA}} k a-h a-r e-e^{\dagger}\right)$-et LXX-iš karl(a-aš p)]é- $\left[\boldsymbol{e}^{\boldsymbol{\eta}}\right.$-ra-an ti-an-zi «[(su, nel tempio del dio della tempesta, dieci gal)]lette venti volte [( si mette)] dav[(anti)] all'altare; [(x boccone di pane di dieci misure? e nove! gallette venti volte)] si met[(te)] accanto al paiolo?; [(tre gallette venti volte il fabbro di Zippa)]landa met[(te)] nei bicchieri. // [(Giù, nel palazzo sei gallette $\mathrm{x})$ ] dieci volte, un pane tunik cinquanta volte, [(1 ${ }^{\text {? }}$ pane kaheret $\left.)\right]$ settanta volte si mette davanti ai gradini»;

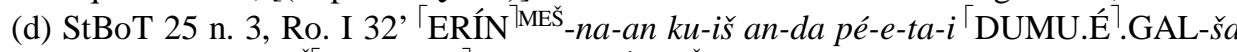
(33’) pé-e-ra-aš-še-et ${ }^{\mathrm{GIS}}{ }^{2}$ zu-pa-a-ri ${ }^{\rceil}$har-zi ERÍN ${ }^{\mathrm{MES}}$-na-an a-ap-pa-an(-)an-da (34') pé-eta-i «davanti a colui che porta dentro le truppe, il paggio tiene una fiaccola $<\mathrm{e}>$ porta dentro le truppe dietro»;

(e) StBoT 17, Ro. 13 nu-uz-za DUMU.NITA ${ }^{\mathrm{MEŠ}}$ kar-ti-iš-mi (14) pé-ra-an me-e-mi-ir «i giovani si dissero (lett.: parlarono davanti al loro cuore)» ${ }^{22}$.

La difficoltà principale che si incontra nel considerare gli avverbi in -an come nominativi accusativi neutri singolari è spiegare come adirezionale la funzione del caso accusativo in dipendenza da verbi di movimento, sia transitivi che intransitivi, che in $\mathrm{OH}$, come già detto, con tutti gli altri nomi assolve all'espressione della direzione ${ }^{23}$ :

(f) StBoT 25 n. 3, Ro. I 3' [III-i]š LUGAL-un MUNUS.LUGAL-an-na hu-ya-an-zi «camminano [tre v]olte verso il re e la regina»;

(g) StBoT 25 n. 31, Vo. III 8' ha-a-aš-ša-an-kán hu-ya-an-zi «camminano verso il focolare»;

20 Questi avverbi possono avere anche una semantica diversa da quella locale, si veda Francia 2002, 43 e segg. et passim.

21 Otten 1973.

22 L'espressione karti (-šmi) peran mema- è stata trattata da Justus 1983, 107 e segg. nota 35; Archi 1995 , 13 e segg.; CHD, P, 305; Francia 2010, 164 e segg.; Pozza 2014, 59 e segg.

23 Hoffner 1997; Francia 1996b, 137 e segg. 
(h) StBoT 25 n. 3, Vo. IV 38 tu-uš al-ki-i[š-t]a-a-an tar-na[-a]h-hé "e li (= gli uccelli) lascio andare verso il ramo»;

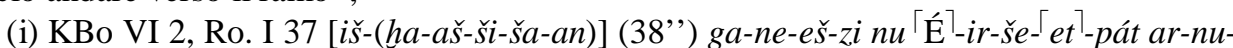
$u z-z i$ «[(il suo) pa(drone lo)] riconosce ed egli alla sua stessa casa $<$ lo $>$ fa giungere» (§ 19a I).

In queste attestazioni il nome in caso accusativo in relazione ai verbi di moto esprime senza alcun dubbio una direzione. Tale evidenza, unitamente alle difficoltà di analizzare come appositivo un sintagma costituito da un avverbio morfologicamente corrispondente ad un nom. acc. n. sing. e un nome in caso in - $i$, dat.loc., come nella costruzione di tipo (1), riteniamo difficile considerare gli avverbi in -an nominativi accusativi neutri singolari. Osservando la loro sintassi, essi sembrano piuttosto doversi spiegare come forme di originari locativi.

\section{PROPOSTA INTERPRETATIVA}

Partendo da osservazioni analoghe, relative alla funzione degli avverbi in -an e, parallelamente dell'avverbio šer, Philo H.J. Houwink ten Cate ha proposto di ritenere queste forme funzionanti come locativi adesinenziali ${ }^{24}$. In un recente contributo Norbert Oettinger sottolinea il carattere locativo degli avverbi ittiti in -an, proprio in quanto opposti funzionalmente alle corrispondenti forme in $-a$, attribuendo dunque la funzione locativa proprio all'-an. Sulla base di questa considerazione, lo studioso propone di analizzare l'ittita andan e, parallelamente, il greco है $\delta \delta o v$, come *endo-n dove la *-n è la variante postvocalica in grado zero della posposizione $*_{-} e^{25}$.

Da un punto di vista meramente formale gli avverbi di luogo corrispondenti ittiti, compreso šer - šara, possono dunque essere ripartiti in tre gruppi:

\begin{tabular}{|c|c|}
\hline$\overline{a p p}-a n$ & $\bar{a} p p-a$ \\
\hline per-an & par- $\bar{a}$ \\
\hline šer & šar-āa \\
\hline
\end{tabular}

Gli avverbi del primo quadrante sono caratterizzati morfologicamente dal raddoppiamento grafico della consonante finale del tema davanti ad -an e, da un punto di vista sintattico, nella lingua antica non sono attestati con il pronome possessivo enclitico suffisso ${ }^{26}$.Dei due avverbi del secondo quadrante, šer è stato riconosciuto da tempo essere

24 Houwink Ten Cate 1966, 127 e segg.: «In my opinion they are neither accusative nor neuter.[ ...] appan, piran and šer function as endingless locatives [...]».

25 Oettinger 2016, 232 e seg.

26 Attestazioni di āppan e kattan in questa costruzione sono relative al solo pronome possessivo di III persona sing. e plur nom. acc. n. -šet /-šmet e sono documentate solo in copie tarde di testi antichi, come sottolinea Starke 1977, 133 nota 9. Le forme āppan-šet /-šmet sono state ritenute in Friedrich-Kammenhuber (1975, 160161) quali esempi di «Volksetymologie» per EGIR- ŠU/ -ŠUNU. 
un «endungslose Lokativ» ${ }^{27}$. Ad esso proponiamo di accostare il tema per- di peran con cui è confrontabile sia per la formazione ${ }^{*} \mathrm{CeR}$, sia per la presenza dell'accento sulla vocale tematica pēran e še $r$, quando occorre con la scriptio plena della vocale tematica $E^{28}$ accanto a cui, tuttavia, già in $\mathrm{OH}$, è attestata anche la scriptio brevis peran, e sarà proprio quest'ultima forma che si affermerà nei periodi posteriori ${ }^{29}$. Dal punto di vista sintattico, una caratteristica comune sia a peran che a šer è la costruzione con il pronome possessivo enclitico nom. acc. n. sing. (-mit, -šit/- šet, -šmit /-šmet) nella lingua antica:

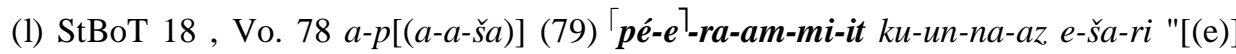
quel[(lo)] siederà davanti a me a destra";

(m) StBoT 25 n. 3, Ro.II 16’ MUŠEN ha-a-ra-na-an L[UGAL-aš SAL.LUGAL-aš-š]a šee-er-ša-me-et III[(-ŠU)] (17’) DUMU.É.GAL wa-ah-nu[-zi "il paggio fa girare per tre [(volte)] l'aquila su di loro, il r[e] e [la regin]a " ;

Anche nella forma direttiva šarā e parā presentano una morfologia del tema pressoché simile: ${ }^{*} \mathrm{C}-\mathrm{a}-\mathrm{r}-$.

L'avverbio andan sintatticamente presenta delle particolarità che suggeriscono di considerarlo a parte rispetto agli altri. Contrariamente a tutti gli altri avverbi, infatti, esso non governa il caso genitivo in funzione posposizionale, ma il dativo locativo già in $\mathrm{OH}$, né è in costruzione con i pronomi possessivi suffisso, come peran e šer. Riguardo alla sua formazione, come già detto, è fatta risalire all'aggiunta del grado zero della posposizione $*_{e n}^{*}$, al tema *endo ${ }^{30}$.

\section{IL «FORMANTE»-an(-)}

La caratteristica che accomuna andan e peran agli avverbi del quadrante superiore (âppan, kattan) è, da un punto di vista morfologico, l'uscita in -an.

Il fatto che šer esprima la nozione della stasi e dell'assenza di direzione, è dovuto proprio alla sua originaria natura di locativo adesinenziale. Negli avverbi corrispondenti, ad eccezione di šer, l'opposizione funzionale adirezionale: direzionale è marcata proprio dall'uscita -an rispetto ad $-a$, è necessario dunque soffermarsi e riflettere sulla natura di questo morfema che, come è evidente alla luce di quanto affermato fino ad ora, non può essere più accostato a quello della desinenza di nom. acc. n. sing. dei temi in - $a$ -

Un elemento -an(-) atto a marcare la stasi è stato individuato da E. Neu quale «formante» nelle forme pronominali in caso dat.loc. apedani, edani, kedani, analizzati come aped-an-i, ed-an-i, ked-an-i ${ }^{31}$, nonché in fine di parola in apadd-an šer ${ }^{32}$. Lo

Neu 1980a, 35 e segg.; si veda anche Oettinger 2000, 185 e segg.

28 Sull'argomento Carruba 1981, 232 e segg.

29 Relativamente alle etimologie proposte per questi avverbi rimandiamo a Rieken 1999, 67-68 con riferimenti alla bibliografia precedente. Per l'accostamento di peran, derivato da un probabile *per, a šer, si veda anche Kloekhorst 2008, 667. La scriptio brevis di šer è attestata con certezza nei testi MH: Francia 2002, passim.

30 Oettinger 2016, 232 e seg.

31 Neu 1974, 67-72.

32 Altrimenti Oettinger 1982, 366. 
studioso dapprima avanza l'ipotesi di confrontare l'-an di āppan, kattan, peran con l'-an(-) di aped-an-i, ed-an-i, ked-an-i, ma appena poche righe dopo, sembra cambiare opinione e passare a considerare gli avverbi adirezionali forme di nominativi accusativi neutri singolari di temi in $-a^{-33}$. Alla base di questa scelta vi è la necessità di spiegare adeguatamente la congruenza grammaticale degli avverbi adirezionali con le forme di nom. acc. n. sing. dei pronomi possessivi enclitici ad essi suffissi.

In uno studio successivo, E. Neu riconosce le forme aped-, ked-, ed- locativi adesinenziali ${ }^{34}$, pertanto le forme pronominali aped-an-i, ked-an-i, ed-an-i risulterebbero costruite sulla base di antichi locativi adesinenziali, ulteriormente confermati nella loro funzione locativa sia dal formante -an(-), già identificato nello studio del 1974, che dalla desinenza nominale del dat.loc.-i.

Una particolarità sintattica del loc. adesinenziale è di indicare tanto un movimento verso un determinato punto, quanto una stasi, ma le forme aped-an-i, ked-an-i, ed-an-i, proprio perché marcate da ben due morfemi per la stasi, l'-an- e la desinenza del dat.loc.-i, risultano essere dei locativi veri e propri, indicanti unicamente la stasi. L'esistenza dell'espressione avverbiale apadd-an šer induce a pensare che in una prima fase i locativi adesinenziali aped-, ked-, ed- furono caratterizzati nella loro funzione stativa dal solo formante -an e, solo successivamente, al momento dell'entrata nella declinazione pronominale, questa funzione venne ulteriormente confermata dalla desinenza del dat.loc. in -i. La recente proposta di N. Oettinger, cui abbiamo già fatto cenno, sembra essere risolutiva a questo proposito, riconoscendo -an derivare dalla posposizione *-en/-n «in» ${ }^{35}$.

Sintatticamente, una delle particolarità dell'avverbio peran è di essere attestato in $\mathrm{OH}$ con forme pronominali enclitiche in $-t /-d$, considerate nominativi accusativi neutri singolari dei pronomi possessivi enclitici. Come già ribadito, è proprio l'occorrenza degli avverbi adirezionali con i pronomi possessivi enclitici uno dei principali argomenti a sostegno della teoria che gli avverbi in -an siano da vedersi quali nominativi accusativi neutri singolari, al pari dei possessivi stessi. I pronomi in $-t /-d$ sono tuttavia attestati anche con šer che, di certo in origine, ma secondo E. Neu ancora nei testi antichi, è un locativo adesinenziale e non un nom. acc. n. sing. ${ }^{36}$

Il primo a mettere in dubbio la natura di possessivi enclitici in caso nom. acc. n. sing. delle forme pronominali in $-t /-d$ suffisse agli avverbi in -an è stato Ph. Houwink ten Cate che ha proposto di vedere in queste forme una funzione locativa: «(they) are therefore followed by the possessive forms ending in - $d$ with a locative function», analogamente a quella degli avverbi in -an a cui sono suffisse ${ }^{37}$.

\footnotetext{
Neu 1974, 67-68.

Neu 1980a, 20.

35 Oettinger 2016, 232 e seg. Precedenti proposte interpretative riguardo ad un formante -an- affisso a locativi adesinenziali specificanti la stasi sono state avanzate da Hajnal 1992, 211 e segg. con bibliografia precedente

37 Houwing ten Cate 1966, 227; si veda anche Francia 1996a: 225-226.; Hoffner - Melchert 2008, 140, 300 relativamente alla distribuzione della desinenza -et/-it del neutro tra forme pronominali e avverbiali.
} 


\section{LA FINALE $-t /-d$ DEI PRONOMI SUFFISSI AGLI AVVERBI ADIREZIONALI}

In ittita vi sono alcuni avverbi di origine pronominale e con funzione di stasi caratterizzati dalla dentale finale $-t /-d$ : i locativi adesinenziali apit e ket, il pronome interrogativo kuwapit oltre alle forme costitutive dei già menzionati aped-an-i, ked-an-i, ed$a n-i^{38}$. La dentale finale $-t /-d$ di queste forme è stata paragonata da alcuni studiosi a quella

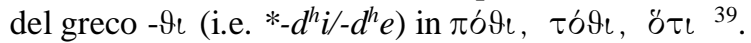

Nello studio da noi condotto sulla declinazione dei pronomi possessivi enclitici, abbiamo proposto di considerare le forme pronominali enclitiche in $-t /-d$ suffisse in $\mathrm{OH}$ a peran e a šer solo formalmente simili a quelle dei pronomi possessivi enclitici suffissi ai nomi neutri ${ }^{40}$. A nostro parere, la dentale finale di queste forme condivide la medesima origine di quella di apit, ket, ed-an-i etc. e degli avverbi greci in -qi. A sostegno di questa ipotesi può avanzarsi anche l'analisi di H.C. Melchert, che individua un diverso vocalismo nelle forme pronominali in - $t$ - $d$ suffisse agli avverbi in -an e a šer rispetto ai nominativi accusativi neutri dei possessivi enclitici suffissi ai nomi neutri ${ }^{41}$.

Non è da trascurare, inoltre, che da un punto di vista sintattico le forme pronominali suffisse agli avverbi siano rimpiazzate dai pronomi personali in caso dat.loc., e non in caso gen., come invece accade per i pronomi possessivi suffissi ai nomi, nelle fasi linguistiche successive all'antica. Stando a queste considerazioni, tra -šet di pedan-šet e -šit di peran-šit potrebbe intercorrere la stessa differenza che vi è tra il pronome neutro in caso nom. acc. $\mathrm{n}$. sing. apat e locativo adesinenziale apit: la dentale finale è solo formalmente identica in epoca storica, derivando l'una (apat) da i.e. *gli-t/-d, e l'altra (apit) da i.e. ${ }^{*}-d^{h} i /-d^{h} e^{42}$. In base a questa analisi, in peran-šit è da vedersi una forma avverbiale caratterizzata da un elemento locativo -an, con funzione stativa in $\mathrm{OH}$, e una forma pronominale con funzione locativa con uscita in $-t /-d$.

\section{CRONOLOGIA DEGLI AVVERBI DI LUOGO CORRISPONDENTI}

In base alle caratteristiche sintattiche proprie degli avverbi di luogo corrispondenti nella lingua antica, è possibile tracciare una sorta di scala cronologica relativa al loro uso:

andan potrebbe essere entrato a far parte del sistema avverbiale ittita già come caso della declinazione + posposizione: ${ }^{*}$ endo $-n,{ }^{43}$. Presumibilmente è questo il più arcaico degli avverbi di luogo ittiti, ritrovandosi con le medesime caratteristiche anche in greco ( semantica e sintattica riassumibili come segue: è documentato all'inizio della frase, sintatticamente indipendente da qualsiasi altro costituente, vale a dire in funzione avverbiale; ha sviluppato già nella lingua antica una semantica che travalica quella strettamente locale, come provato dal significato «inoltre», individuabile in taluni contesti

\footnotetext{
38 Gli avverbi pronominali sono stati analizza da Josephson 1966, 133 e segg.; per i locativi adesinenziali si veda Neu 1980a, 20 e segg.

39 Szemerényi 1956, 57 e segg.; Eichner 1974, 34; Krisch 1984, 132-133.

40 Francia 1996a, 226.

41 Melchert 1984, 123.

42 Neu 1980a, 22 e segg., 50 e seg.

43 Si veda nota 6 per i riferimenti bibliografici.
} 
nella forma direttiva corrispondente $a d^{44}$; non sono attestate costruzioni con pronomi suffissi;

peran fu formato probabilmente partendo da un originario locativo adesinenziale, *per, non più presente come tale nel vocabolario ittita, ma le cui tracce restano nel greco $\pi \varepsilon \hat{\rho} \rho$ nel latino $\mathrm{per}^{45}$, la cui funzione di stasi in epoca storica è ulteriormente marcata dal formante locativale -an. È questo l'unico avverbio corrispondente che, oltre alla costruzione con il caso gen., tipica della lingua antica, mostra anche quella con il caso dat.loc. (-i), tipica delle costruzioni posposizionali delle fasi posteriori. Questa doppia possibilità di costruzione potrebbe testimoniare una relativa lunga permanenza dell'avverbio peran nella lingua, tanto da innovare nella scelta della reggenza del caso del nome da esso governato prima degli altri avverbi, che infatti nei testi antichi sono in relazione al solo caso gen. Contrariamente ad appan e kattan, già in $\mathrm{OH}$ è in costruzione con le forme pronominali enclitiche in -t-/-d (peran-šit);

šer è un originario locativo adesinenziale. La forma caratterizzata dalla particella -an è individuabile nella particella locale di inizio frase -šan, derivata da *ša(r)-an ${ }^{46}$. Nella lingua antica šer è in relazione al caso dat.loc. (-i) ed è costruito con le forme pronominali

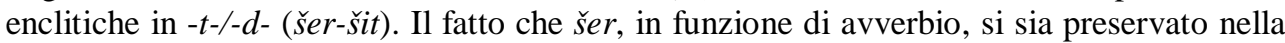
originaria forma di loc. adesinenziale, unitamente alla sua occorrenza come posposizione ai soli nomi in caso dat.loc., potrebbe indurre a collocare la sua entrata nel gruppo avverbiale ittita in uno stadio relativamente più tardo rispetto a quello di peran;

appan e kattan si formarono probabilmente in un momento successivo, in analogia a peran e all'assonante andan, già presenti al momento della loro entrata. A favore della relativa recenziorità di questi avverbi parlano sia le costruzioni sintattiche in cui sono documentati, che la loro stessa morfologia. Da un punto di vista morfologico, essi non possono essere ritenuti forme di locativi adesinenziali, né sintatticamente ne presentano le caratteristiche. Già nella lingua antica, infatti, non occorrono in costruzione con le forme pronominali enclitiche in $-t /-d$-, anzi dal tema katt- è formato katti-, verosimilmente proprio per la costruzione con gli elementi pronominali, ma in caso dat.loc. i: katti-ši. Per la costruzione posposizionale di appan e kattan, probabilmente in analogia a peran, si ricorse a nomi in caso gen.

\section{BIBLIOGRAFIA}

ARCHI, A.

1995 'Pensavano’ gli Ittiti?: Studi Epigrafici e Linguistici 12 (1995), pp. 13-19.

BOLEY, J.

1985a Hittite and Indo-European Place Word Syntax: Die Sprache 31 (1985), pp. 229-241.

1985b Notes on Hittite Place Word Syntax: Hethitica VI (1985), pp. 5-43.

44 Francia 2002, 121.

45 Rieken 1999, 68 con bibliografia precedente.

46 Carruba 1969, 35 e seg.; la particella -šan presenta la stessa sintassi di šer: costruzione con verbi di stato con locativi. 
BRIXHE, C.

1979 Le directif du vieux-hittite et son ascendance indoeuropéenne: Florilegium Anatolicum: CARRUBA, O.

Mélanges offerts à Emmanuel Laroche, Paris, pp. 65-77.

1969 Die Satzeinleitenden Partikeln in den Indogermanischen Sprachen Anatoliens (Incunabula Greaca 32), Roma 1969.

1981 Pleneschreibung und Betonung: Zeitschrift für Vergleichende Sprachforschung 95 (1981), pp. 232-247.

CARRUBA, O. - SOUČEK, Vl. - STERNEMANN, R.

1965 Kleine Bemerkungen zur jüngsten Fassung der hethitischen Gesetze: Archiv Orientálí 33 (1965), pp. 1-18.

CHD, L-N / P = GÜterbock, A.G. - Hoffner, H.A. (eds.) 1980-: The Hittite Dictionary of the Oriental Institute of Chicago. Chicago.

DUNKEL, G.E.

1992 Die Grammatik der Partikeln: R.S.P. BeEKes - A. Lubotsky - J. WeitenBerg (eds.), Rekonstruktion und Relative Chronologie. Akten der VIII. Fachtagung der Indogermanischen Gesellschaft. Leiden 31. August - 4. September 1987, Innsbruck 1992, pp. 153-177.

EICHNER, $\mathrm{H}$.

$1974 \quad$ Untersuchungen zur Hethitischen Deklination, Dissertation Teildruck, Erlangen 1974.

FRANCIA, R.

1996a Il pronome possessivo enclitico in antico ittita: alcune riflessioni: Vicino Oriente 10 (1996), pp. 209-259.

1996b Funzioni sintattiche nei testi dell'antico Hittita: il locativo di meta e di scopo e l'accusativo di relazione: Incontri Linguistici 19 (1996), pp. 137-153.

1997 L'accusativo nello spazio e nel tempo in ittita: Annali del Seminario di Studi del Mondo Classico, Istituto Universitario Orientale di Napoli 19 (1996), pp. 139-145.

2002 La Funzione Sintattica degli Elementi Avverbiali di Luogo Ittiti: anda(n), āppa(n), katta(n), katti-, peran, parā, šer, šara (Studia Asiana 1), Roma 2002.

2010 Ittita āppa “(via) da”: Incontri Linguistici 33 (2010), pp. 161-166.

2012 Lineamenti di Grammatica Ittita. Seconda Edizione (Studia Asiana 8), Roma 2012.

FRIEDRICH, J. - KAMMENHUBER, A.

1975 Hethitisches Wörterbuch. Zweite völlig neuebearbeitete Auflage auf der Grundlage der edierten hethitischen Texte, Heidelberg 1975.

HAJNAL, I.

1992 Griechisch $\chi \alpha \mu \alpha \iota \alpha$ - ein Problem der Rekonstruktion?: R.S.P. BEEKES - A. LUBOTSKY - J. WEITENBERG (eds.), Rekonstruktion und Relative Chronologie. Akten der VIII. Fachtagung der Indogermanischen Gesellschaft, Leiden 31. August - 4. September 1987, Innsbruck 1992, pp. 207-220.

HOFFNER, H. A. JR.

1997 The Laws of the Hittites, Leiden - New York - Köln 1997.

HOFFNER, H. A. JR. - MELCHERT, H. C.

2008 A Grammar of the Hittite Language. Part 1: Reference Grammar. (Languages of the Ancient Near East), Winona Lake, Indiana 2008.

Houwink Ten Cate, Ph. H.J.

1966 The Ending -d of the Possessiv Pronoun: Revue Hittite et Asianique XXVI/79 (1966), pp. 123-132. 
JOSEPHSON, V.

1966 Pronominal Adverbs of Anatolian Formation and Function: Revue Hittite et Asianique XXVI/79 (1966), pp. 133-154.

1981 Recensione a F. STARKE, Die Funktionen der dimensionalen Kasus und Adverbien im Althethitischen (Studien zu den Bogazköy-Texten 23), Wiesbaden 1977: Kratylos 26 (1981), pp. 95-105.

Justus, C.

1983 Semantic and Syntact Aspects of 'Knowing in one’s Heart’: Orientalia 52, pp. 107-115.

KAMMENHUBER, A.

1979 Direktiv, Terminativ und/oder Lokativ im Hethitischen: E. NEU - W. MEID (eds.), Hethitisch und Indogermanisch: Vergleichende Studien zur historischen Grammatik und zur dialektgeographischen Stellung der indogermanischen Sprachgruppe Altkleinasiens (Innsbrucker Beiträge zur Sprachwissenschaft 25), Innsbruck 1979, pp. 115-142.

KLOEKHORST, A.

2008 Etymological Dictionary of the Hittite Inherited Lexicon. (Leiden Indo-European KRISCH, R. Etymological Dictionary Series 5), Leiden 2008

1984 Konstruktionsmuster und Bedeutungswandel indogermanischer Verben. Anwendugsversuche von Valenztheorie und Kasusgrammatik auf Diachronie und Rekonstruktion, Frankfurt a.M. - Bern - New York - Nancy 1984.

MELCHERT, H.C.

1984 Studies in Hittite Historical Phonology (Ergänzungshefte zur Zeitschrift für Vergleichende Sprachforschung 32), Göttingen 1984

1994 Anatolian Historical Phonology, Amsterdam-Atlanta, GA 1994.

NEU, E.

1974 Der Anitta Text (Studien zu den Bogazköy-Texten 18), Wiesbaden 1974.

1980a Studien zum endungslosen "Lokativ" des Hethitischen (Innsbrucker Beiträge zur Sprachwissenschaft Vorträge und kleiner Schriften 23), Innsbruck 1980.

1980b Althetische Ritualtexte in Umschrift (Studien zu den Bogazköy-Texten 25), Wiesbaden 1980.

OETTINGER, N.

1982 recensione a E. NEU Studien zum endungslosen "Lokativ" des Hethitischen. (Innsbrucker Beiträge zur Sprachwissenschaft Vorträge und kleiner Schriften 23), Innsbruck 1980: Bibliotheca Orientalis 39 (1982), pp. 364-368.

2000 Hethitisch šser "auf": J. ARBeitman (ed.), The Asia Minor Connexion. Studies in Memory of Charles Carter, Leuven - Paris 2000, pp. 185-188.

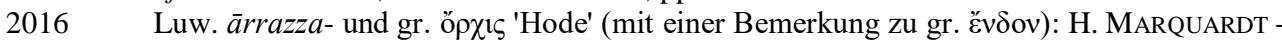
S. Reichmuth - J. V. García Trabazo (eds.), Anatolica et Indogermanica. Studia linguistica in honorem Johannis Tischler septuagenarii dedicata. Innsbruck 2016, pp. 231-240.

OTTEN, $\mathrm{H}$

1973 Eine althetitische Erzählung um die Stadt Zalpa (Studien zu den Bogazköy-Texten 17), Wiesbaden 1973.

PozzA, M.

2014 Itt. išta(n)h- e mema/i-: 'esperire' e 'riflettere' tra concretezza e metafora: Rivista degli Studi Orientali N.S. 87 (1-4) 2014, pp. 57-72.

RIEKEN, E.

1999 Untersuchungen zur nominalen Stammbildung des Hethitischen (Studien zu den Bogazköy-Texten 44), Wiesbaden 1999. 
SALISBURY, D.

$1999 \quad$ anda and andan in Neo-Hittite: Journal of Cuneiform Studies 51 (1999), pp. 61-72.

STARKE, F.

1977 Die Funktionen der dimensionalen Kasus und Adverbien im Althethitischen (Studien zu den Bogazköy-Texten 23), Wiesbaden 1977.

SZEMERÉNYI, O.

1956 Hittite Pronominal Inflection and the Development of Syllabic Liquids and Nasals: Zeitschrift für Vergleichende Sprachforschung 73 (1956), pp. 57-80.

TJERKSTRA, F.A.

1999 Principles of the Relation between Local Adverb, Verb and Sentence Particle in Hittite (Cuneiform Monographs 15), Groningen 1999. 